\title{
926.
}

\section{CORRECTED SEMINVARIANT TABLES FOR THE WEIGHTS 11 AND 12.}

[From the American Journal of Mathematics, vol. xIv. (1892), pp. 195-200.]

ThE tables in my paper, "Seminvariant Tables," American Journal of Mathematics, vol. vII. (1885), pp. 59-73, [831], are not in the best form, but the deviations present themselves only in a few columns of the tables for the weights 11 and 12, viz. in the former of these two columns, and in the latter a single column, ought to be replaced by linear combinations of other columns; there are, besides, columns which should be new named; in regard hereto, there is a point of theory which requires to be made clear. I remark that in each table the literal terms are in alphabetical order $(A O)$; this is the proper order for the final terms, and although (as about to be explained) the proper order for the initial terms is the counter order $(C O)$, yet as the tables cannot be at the same time arranged in the one and the other order, I adhere to the $A O$ as the proper arrangement for the terms of the tables; we have, however, to introduce the notion that, in general, it is not the top term of a column which is to be regarded as the initial term, and in connexion herewith to consider how the columns are to be named. An instance first presents itself for the weight 11: we have, see column 5 of the table for that weight here given, a seminvariant

$$
\begin{aligned}
& f g+1 \\
& b^{2} j \\
& b c i \\
& b d h \\
& b e g-5 \\
& b f^{2}=6 \\
& c^{2} h-16 \\
& \vdots \\
& b^{3} e^{2}+70
\end{aligned}
$$

C. XIII. 
this is to be regarded, not as a seminvariant $f g-b^{3} e^{2}$ (it is hardly necessary to remark that, here and elsewhere the - is not a minus sign, but a mere stroke), but as a seminvariant $c^{2} h-b^{3} e^{2}$, viz. $c^{2} h$ is a term entering into the seminvariant, and which, although it is in $A O$ subsequent, it is in $C O$ precedent, to the terms $f g$, beg and $b f^{2}$. The seminvariant contains the term $-16 c^{2} h$ and other terms with the letter $h$, and it is a misnomer to call it $f g-b^{3} e^{2}$, a name implying that the highest letter thereof is $g$. Instead of the stroke, it would perhaps be better to write $\infty$, for instance $c^{2} h \infty b^{3} e^{2}$, where of course $\infty$ would be used as a mere conventional symbol.

For greater clearness, I give here the express definition of counter order, $(C O)$, viz. whereas in $A O$ we begin with the lowest letters, in $C O$ we begin contrariwise with the highest letters. A term containing a higher letter or higher power of such letter precedes a term containing a lower letter or lower power of the same letteror in the easiest form, the counter order is the alphabetical order corresponding to the reversed arrangement $z, y, \ldots, f, e, d, c, b$ of the letters.

A symbol, as $c^{2} h-b^{3} e^{2}$ above, may be regarded as referring to a set of terms $c^{2} h, b^{3} e^{2}$ and all the terms which are in $C O$ subsequent to $c^{2} h$ and in $A O$ precedent to $b^{3} e^{2}$ : as by supposition the terms are arranged in $A O$, the set includes no term lower than $b^{3} e^{2}$, or say the bottom term $b^{3} e^{2}$ is also the final term of the set, but it does include terms $f g$, beg and $b f^{2}$ higher than $c^{2} h$, and thus the top term $f g$ is not, but $c^{2} h$ is, the initial term of the set. It should be remarked that a seminvariant $c h-b^{3} e^{2}$ need not include all the terms of the set as just defined: there may very well be terms with a coefficient zero, or say accidental zeros; an instance presents itself, weight 10 , where in the column $e g-b d^{3}$ we have $0 c e^{2}$, no term in $c e^{2}$.

The changes actually required are very slight, viz.

Weight 11, instead of

$$
\begin{array}{lll}
f g-b^{3} e^{2}, \text { we require } & c^{2} h-b^{3} e^{2}, \text { old } f g-b^{3} e^{2}, \text { new named, } \\
c^{2} h-b^{3} d^{2}, & \# \quad f g-b^{3} d^{2}, \text { linear combination }\left(f g-b^{3} e^{2}\right)+8\left(c^{2} h-b^{3} d^{2}\right), \\
d e^{2}-b^{5} d^{2}, & \text { " } & c^{3} f-b^{5} d^{2}, \text { old } d e^{2}-b^{5} d^{2}, \text { new named, } \\
c^{3} f-b^{3} c^{4}, & \# \quad d e^{2}-b^{3} c^{4}, \text { linear combination }\left(d e^{2}-b^{5} d^{2}\right)+6\left(c^{3} f-b^{3} c^{4}\right) .
\end{array}
$$

Weight 12 , instead of

$$
\begin{aligned}
& c f^{2}-b c d^{3}, \text { we require } d^{2} g-b c d^{3}, \text { old } c f^{2}-b c d^{3}, \text { new named, } \\
& d^{2} g-c^{3} d^{2}, \quad \quad, \quad c f^{2}-c^{3} d^{2}, \text { linear combination }\left(c f^{2}-b c d^{3}\right)-5\left(d^{2} g-c^{3} d^{2}\right)
\end{aligned}
$$

but I have thought it desirable to give the complete tables for the weights in question, 11 and 12; and I have also rearranged the entire columns of the two tables so as to present in each of them the finals in AO. This is the case with the existing tables, except that there is a single transposition in the table weight 10 . 
926] CORRECTED SEMINVARIANT TABLES FOR THE WEIGHTS 11 AND 12.219

Instead of the columns $c d f-b^{4} d^{2}, c e^{2}-c^{5}$, we ought to have $c e^{2}-c^{5}, c d f-b^{4} d^{2}$. The complete list up to the weight 12 is

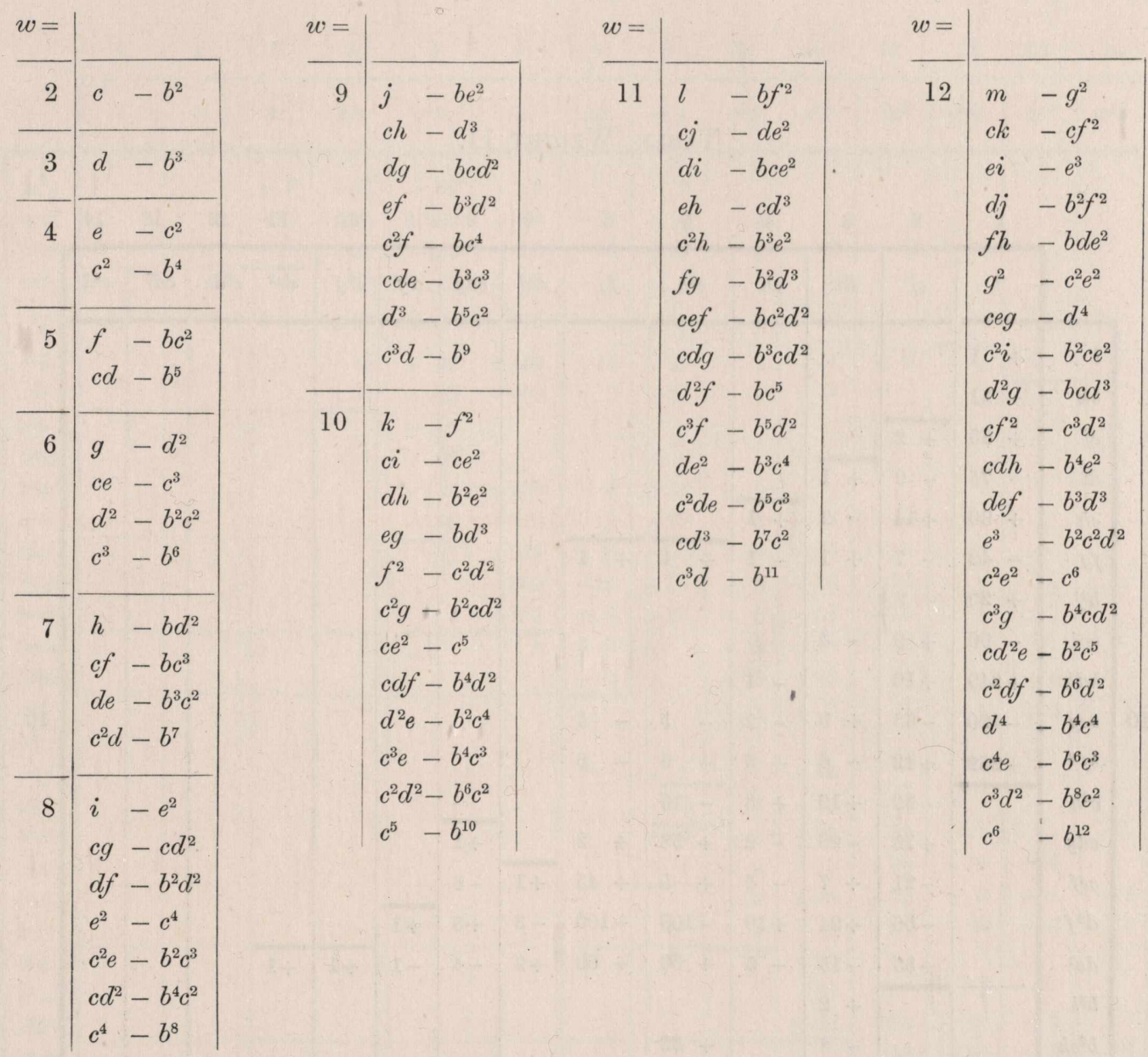

The two new tables are as follows: some accidental numerical errors have been corrected. 
Table, Weight 11.

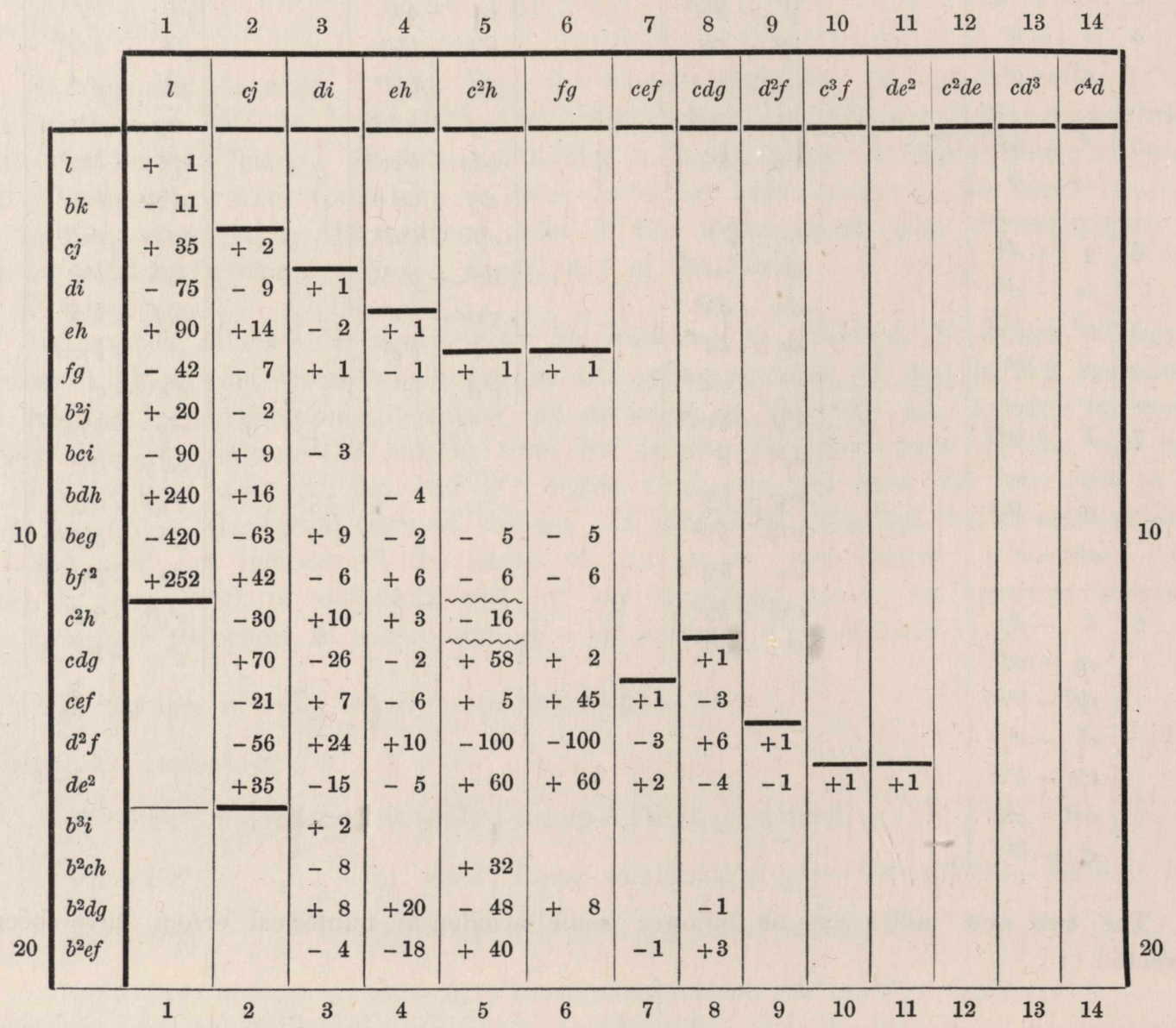


Table, WeIght 11 (continued).

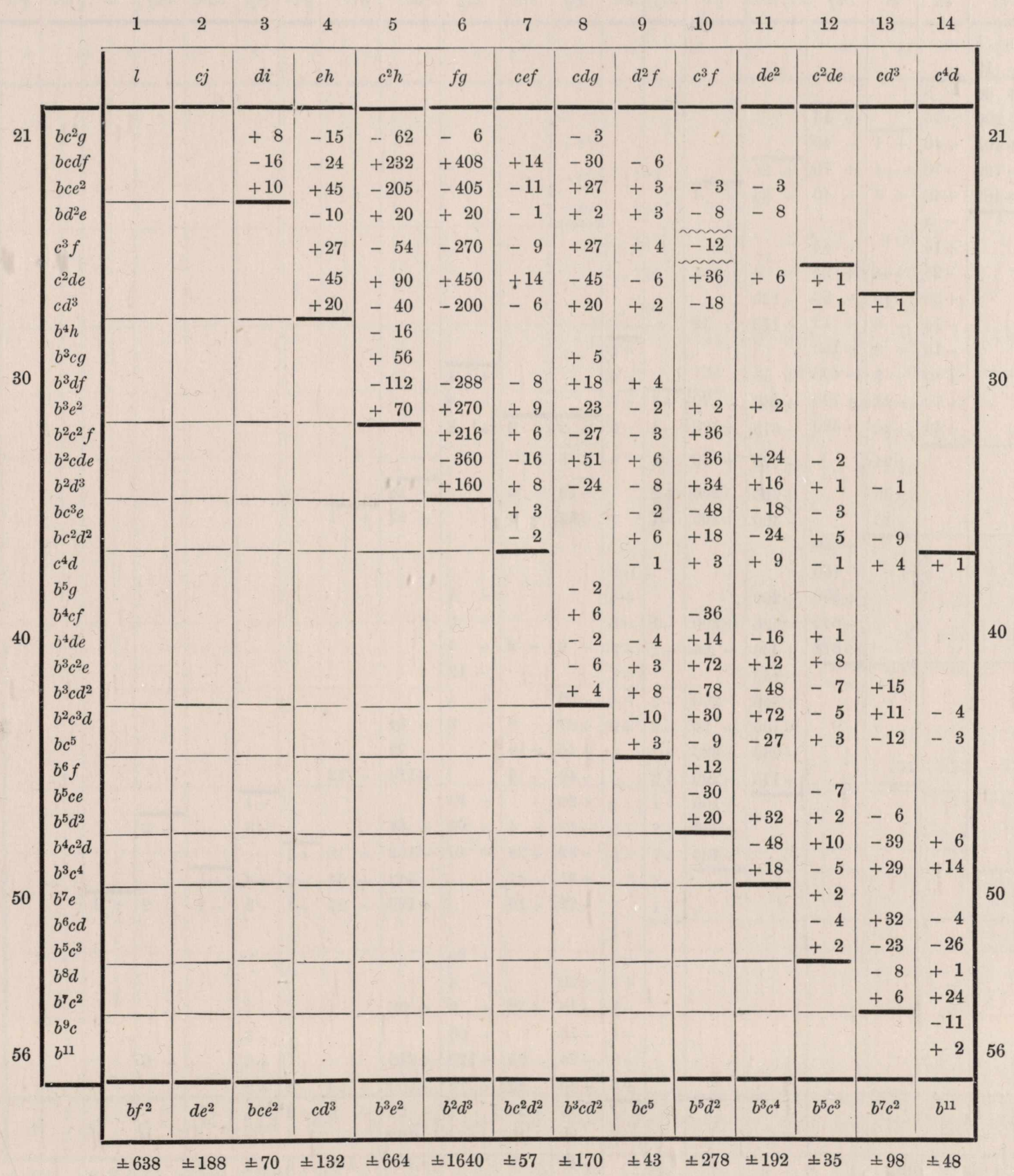


Table Weight 12.

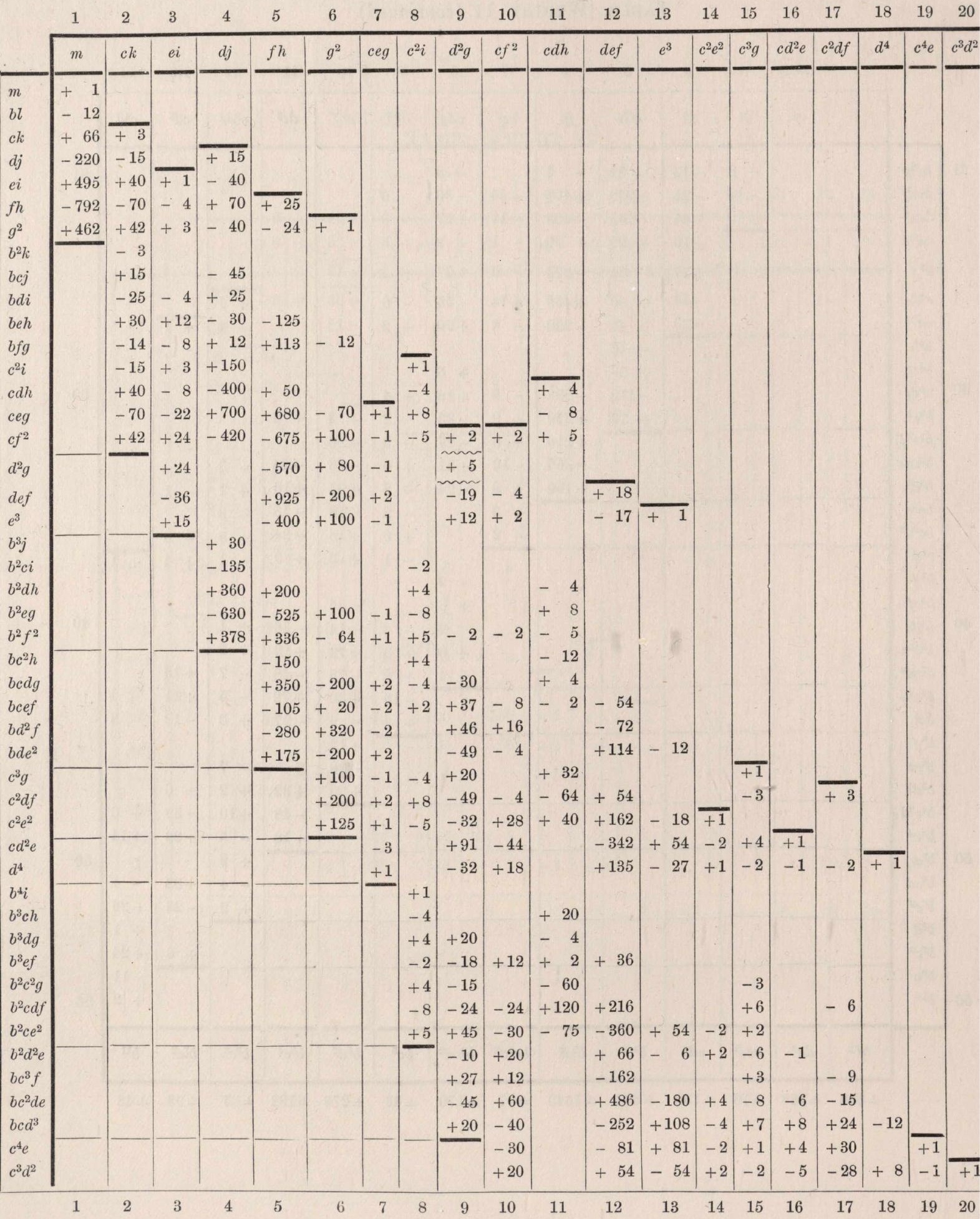


TABLe, Weight 12 (continued).

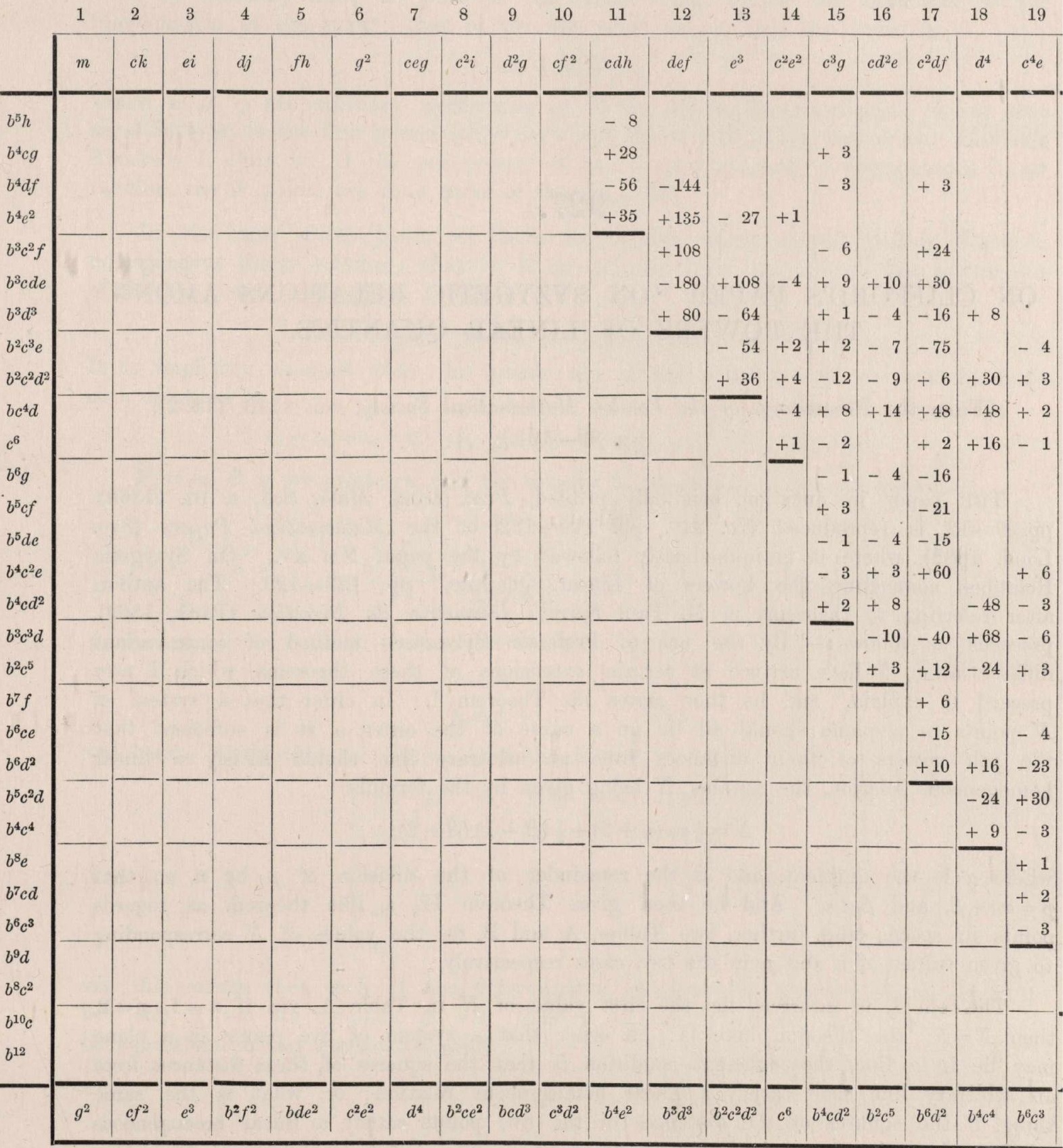

$\pm 1024 \pm 212 \pm 82 \pm 1740 \pm 2854 \pm 946 \pm 12 \pm 46 \pm 325 \pm 190 \pm 298 \pm 1664 \pm 442 \pm 18 \pm 52 \pm 51 \pm 258 \pm 156 \pm 48$ 